\title{
Correlation Imaging Reveals Specific Crowding Dynamics of Kinesin Motor Proteins
}

\author{
Daniël M. Miedema, ${ }^{1,2, *}$ Vandana S. Kushwaha, ${ }^{3}$ Dmitry V. Denisov, ${ }^{1}$ Seyda Acar, ${ }^{3}$ \\ Bernard Nienhuis, ${ }^{1}$ Erwin J. G. Peterman, ${ }^{3}$ and Peter Schall ${ }^{1}$ \\ ${ }^{1}$ Institute of Physics, University of Amsterdam, P.O. Box 94485, 1090 GL Amsterdam, Netherlands \\ ${ }^{2}$ Laboratory for Experimental Oncology and Radiobiology (LEXOR), \\ Center for Experimental Molecular Medicine (CEMM), Academic Medical Center, \\ Meibergdreef 9, 1105 AZ, Amsterdam, Netherlands \\ ${ }^{3}$ Department of Physics and Astronomy and LaserLaB, Vrije Universiteit, \\ 1081 HV Amsterdam, Netherlands
}

(Received 20 March 2017; revised manuscript received 24 August 2017; published 16 November 2017)

\begin{abstract}
Molecular motor proteins fulfill the critical function of transporting organelles and other building blocks along the biopolymer network of the cell's cytoskeleton, but crowding effects are believed to crucially affect this motor-driven transport due to motor interactions. Physical transport models, like the paradigmatic, totally asymmetric simple exclusion process (TASEP), have been used to predict these crowding effects based on simple exclusion interactions, but verifying them in experiments remains challenging. Here, we introduce a correlation imaging technique to precisely measure the motor density, velocity, and run length along filaments under crowding conditions, enabling us to elucidate the physical nature of crowding and test TASEP model predictions. Using the kinesin motor proteins kinesin-1 and OSM-3, we identify crowding effects in qualitative agreement with TASEP predictions, and we achieve excellent quantitative agreement by extending the model with motor-specific interaction ranges and crowding-dependent detachment probabilities. These results confirm the applicability of basic nonequilibrium models to the intracellular transport and highlight motor-specific strategies to deal with crowding.
\end{abstract}

DOI: 10.1103/PhysRevX.7.041037

\section{INTRODUCTION}

Living cells require a constant supply of energy to maintain their highly organized structures and specialized functions. Specific "motor" proteins within the cell consume chemical energy to work against entropic forces, transporting building blocks against concentration gradients. Motor proteins of the kinesin, myosin, and dynein superfamilies navigate by moving along the network of biopolymers of the cytoskeleton, which spans the volume of the cell [1]. These cytoskeleton filaments can be densely populated with motor proteins and other microtubuleassociated proteins. The resulting crowding effects are thought to play a crucial role in the intracellular transport, hampering the motion of the motors, and affecting their run length along the filaments [2]. Yet, how motor-motor interactions affect their progression and collective transport along densely populated filaments remains unresolved.

Simple stochastic microscopic models for out-ofequilibrium dynamics such as the totally asymmetric simple exclusion process (TASEP) [3-8] have been used

\footnotetext{
*Corresponding author. d.m.miedema@amc.nl

Published by the American Physical Society under the terms of the Creative Commons Attribution 4.0 International license. Further distribution of this work must maintain attribution to the author(s) and the published article's title, journal citation, and DOI.
}

Subject Areas: Biological Physics, Statistical Physics

to model this collective motor transport. In its easiest form, TASEP describes the unidirectional progression of particles along a one-dimensional lattice, on which each particle can proceed as long as the target site is empty but cannot proceed otherwise, as illustrated in Fig. 1. This simple model leads to crowding effects due to the hardcore exclusion of neighboring motors. To include the experimentally observed detachment and attachment of motors to the filament, the model has been extended with Langmuir kinetics (LK) $[9,10]$, in which the motors' unbinding and rebinding to an empty site are described by the rates $\omega_{A}$ and $\omega_{D}$, as shown in Fig. 1(b). Together, $\omega_{A}$ and $\omega_{D}$ control the density of motors on the filament; the resulting density-dependent velocity and run length are sketched in Fig. 1(c). Both decrease and eventually vanish at high density because the particles' exclusion interactions impede their progression. While this model provides a paradigm description of nonequilibrium transport, experimental measurement of real crowded molecular motor transport remains challenging. At high densities, the individual motors can no longer be resolved optically, and the quantitative nature of motor protein crowding remains unclear.

Here, we adopt image correlation techniques to directly measure the density, velocity, and run length of dense moving molecular motors, allowing direct quantitative assessment of crowding effects. We accurately measure the crucial motility parameters of motor protein transport at high density and quantitatively assess TASEP-LK model 
(a)

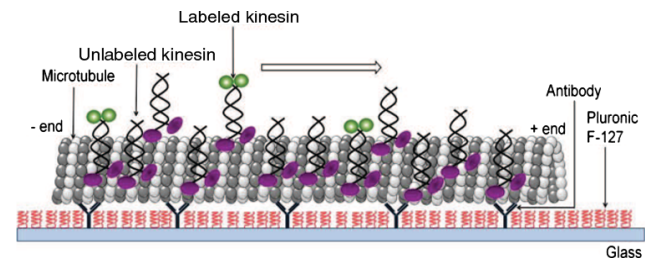

(b)

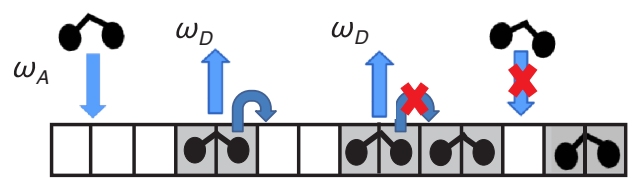

(c)

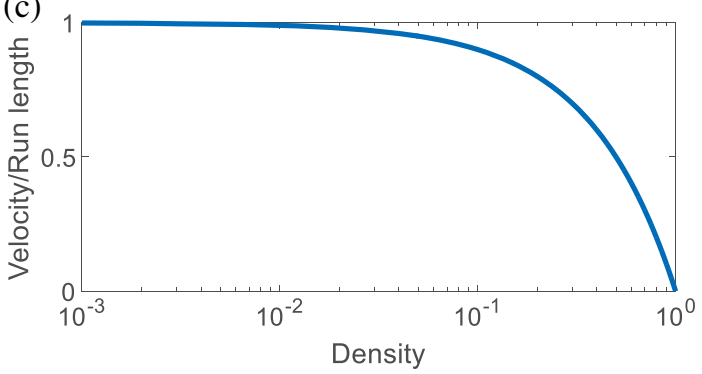

FIG. 1. Crowding of motor proteins along microtubules. (a) Schematic of the in vitro assay. Molecular motors walk along the microtubules attached to the glass slide. The fluorescently labeled motor proteins are excited and imaged using TIRF microscopy from below. (b) TASEP-LK model. The schematic illustrates the attachment, progression, and detachment of the motors. Motors attach to unoccupied lattice sites with rate $\omega_{A}$ and detach from the lattice with rate $\omega_{D}$. Each motor occupies two lattice sites (indicated by grey shaded area) and makes steps of one lattice site. (c) Normalized velocity and run length as a function of density as predicted by the TASEP-LK model.

predictions. We focus on two motor proteins: Drosophila melanogaster kinesin-1, the prototypical kinesin that transports cargo in axons towards the axonal tip [11], and Caenorhabditis elegans homodimeric kinesin-2, OSM-3, a motor involved in intraflagellar transport. Both are known to move in well-defined steps of $d=8 \mathrm{~nm}$, the length of a tubulin dimer along the microtubule [12]. Investigating these two types of motors, and comparing with TASEP predictions, we identify motor-specific interaction ranges and detachment probabilities and find that extension of the TASEP-LK model to include parallel lanes of motors, as well as enhanced detachment of pausing motors, is necessary to fully describe the experimental data. These results yield important insight into the nature of motor-specific interactions in crowded molecular motor transport.

\section{CORRELATION IMAGING TECHNIQUE}

We developed a correlation imaging technique to accurately measure the average motor velocity and run length at high densities from images of fluorescently labeled moving motors. The approach is similar to that in particle image

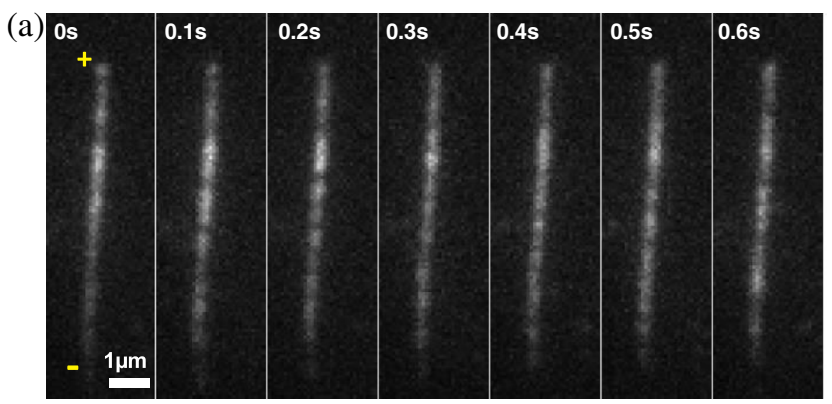

(b)
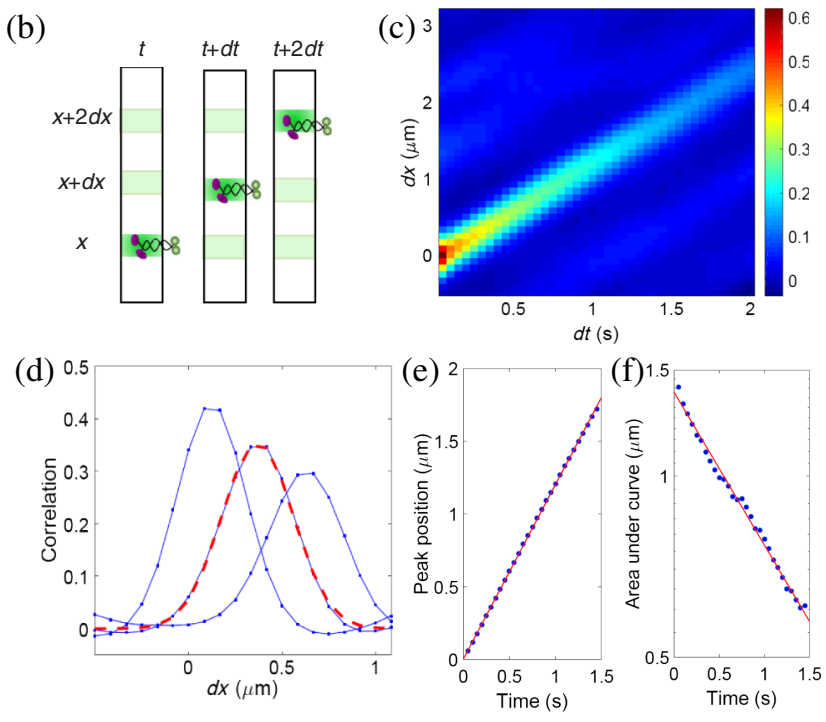

FIG. 2. Correlation imaging measurements at high density. (a) Time series of fluorescently labeled OSM-3 motor proteins on a microtubule at a concentration of $40 \mathrm{nM}$ in PEM12 buffer. The plus and minus ends of the microtubule are indicated. (b) Schematic of the correlation method used to determine the average motor velocity and run length. Fluorescent intensities are correlated in time and space along the microtubule. (c) Spatiotemporal map of correlation of the intensity of the complete image sequence (1000 images) shown in panel (a). Values of high correlation lie along a straight line, the slope of which represents the average velocity of the motors. (d) Cross sections of the correlation surface in panel (c) at delay times $\mathrm{dt}=0.1 \mathrm{~s}, 0.3 \mathrm{~s}$, and $0.5 \mathrm{~s}$. Gaussian fitting (dashed red line) is used to obtain the peak position and area under these curves. (e) Peak position as a function of time. The red linear fit yields the motor velocity. (f) Area under the curve as a function of time in the semilogarithmic representation. The red exponential fit yields the detachment rate of motors.

velocimetry [13-15]: we correlate image intensities in space and time along a microtubule [Fig. 2(a)], using the fact that fluorescent intensities of motors moving with velocity $v=$ $\Delta x / \Delta t$ show strong correlation when correlated over just these space and time intervals, as illustrated in Fig. 2(b). Like in previous studies, the fluorescently labeled motors walking on microtubules attached to the cover slip are imaged using total-internal reflection fluorescence (TIRF) microscopy [Fig. 1(a)]. This technique substantially reduces out-of-focus background fluorescence due to motors that are free in solution. To reduce photobleaching, we use an oxygen 
scavenging system. To ensure steady-state molecular motor transport, we start measurements several minutes after preparing the motility assays; this time is much larger than the inverse attachment and detachment rates of the motors. Furthermore, the ratio of motor proteins versus microtubule binding sides is sufficiently high to avoid depletion effects [16]. We also make sure the motors do not run out of fuel during the measurement by using sufficiently saturated Adenosine triphosphate (ATP) concentrations. In a previous study of crowding on microtubules [8], super-processive kinsesins were studied, which formed jams on microtubule ends. Kinesin-1 and OSM-3, on the other hand, are not as processive, and crowding can be observed all along the microtubule at high motor densities. We thus study motor protein crowding on the bulk of microtubules in a steady state (Fig. S1 in Ref. [17]) by correlation imaging. Although single-particle tracking methods can be applied to measure the velocity and run length when the motors are well separated, these methods break down when the average distance between motors approaches the diffraction limit [Fig. 2(a)], while correlation imaging is still able to track the motion with high accuracy.

The resulting space-time intensity correlation functions of OSM-3 motors indeed reveal a line of positive correlation as shown in Fig. 2(c). The slope of this line gives the average velocity [14] of the motors, which we find to be in excellent agreement with particle tracking results at low density, where single-particle tracking can be applied (Fig. 2 in Ref. [17]). The decay of correlations along this line indicates fluctuations in the motor velocity and number. By accurately tracing the autocorrelation peak using Gaussian fitting [Fig. 2(d)], we determine the average velocity $v=d x / d t$ [Fig. 2(e)], and from the evolution of the area $A$ under the curve [Fig. 2(f)], we determine the rate of motor detachment from the microtubule. We note that while, in principle, any change in local intensity gives rise to a change in the area under the autocorrelation curve, the detachment of fluorescent motors dominates the loss of correlation (see Appendix B). We find that $A$ decays exponentially with time, indicating a constant probability of detachment of the motors. Using the exponential fit $A \propto$ $\exp \left(-\omega_{D} d t\right)$ [Fig. 2(f), red line], we obtain the average run time $\tau=1 / \omega_{D}$ and run length $\lambda=v / \omega_{D}$. We again verify our results by comparison with single-particle tracking at low densities. We find good agreement of both velocity and run length (Fig. 2 in Ref. [17]). We also verified that latter agreement is robust over a large range of fluorescent motor concentrations by varying the ratio of labeled-to-unlabeled motors at constant total motor concentration (see Fig. 2 in Ref. [17]). Even where single-particle tracking can be applied (Fig. 3 in Ref. [17]), correlation imaging is about 10 times faster than standard particle tracking to measure the velocity and run length, making correlation imaging an attractive tool when large amounts of data need to be processed.
To attain even higher densities, we added increasing fractions of unlabeled motors to a fixed amount of labeled motors, as in previous studies $[18,19]$. Since the unlabeled motors otherwise have properties identical to the labeled ones, their affinity to the microtubule is the same, and the ratio of labeled motors in solution and on the filament should not change. We nevertheless observe that the density of labeled motors on the microtubule decreases when we add many unlabeled motors to the solution [Fig. 3(a) and Fig. 4 in Ref. [17]] and interpret this as crowding-related effects in the competition for space on the microtubule. To quantify these density effects and connect the measured velocity and run length to physical transport models, we measure the motor density on the filament directly. We use an approach inspired by fluorescence correlation spectroscopy [20] that measures the number of fluorescent particles in a volume from the fluctuations of the total, spatially integrated, fluorescence intensity [Fig. 3(b)]. Essentially, a small number of fluorescing particles results in large intensity fluctuations, while a larger number of fluorescent objects results in relatively smaller fluctuations, allowing us to measure the number of labeled particles directly from the fluctuation magnitude relative to the mean of the total filament-integrated intensity. The integrated intensity across the microtubule exhibits a Poisson distribution [Fig. 3(c), inset]. Its normalized variance is related to the average number $N$ of fluorescent motors according to $\sigma_{I} /\left(I-I_{B}\right)=1 / \sqrt{N}$, where $I$ is the mean intensity and $I_{B}$ is the time-averaged background intensity in the vicinity of the microtubule. The latter corrects for background-related artifacts. From the number of motors and the length $L$ of the microtubule, we then determine the density $\rho=N / L$ of motors.

The resulting motor density on the filament as a function of motor concentration in solution is shown in Fig. 3(c), where we also indicate manually counted densities as a consistency check (grey shading for kinesin-1). At low motor concentration, the filament density increases in proportion to concentration in solution as expected. At higher concentration, however, the growth flattens, indicating that the limited space no longer allows the accommodation of all motors. Interestingly, deviation from linearity sets in at much lower density for kinesin-1 motors than for OSM-3. For the former, this deviation occurs already for a few motors per micrometer, corresponding to an average spacing of a few hundred nanometers, much larger than their physical size of around $16 \mathrm{~nm}$. For OSM-3 motors, on the other hand, the linear relation continues to almost a hundred motors per micrometer, corresponding roughly to a single fully occupied lane of motors. These density measurements along crowded filaments already reveal remarkably different crowding behavior of kinesin-1 and OSM-3 motors.

We can now plot the velocity and run length of kinesin-1 and OSM-3 motors as a function of motor density on the 
(a)

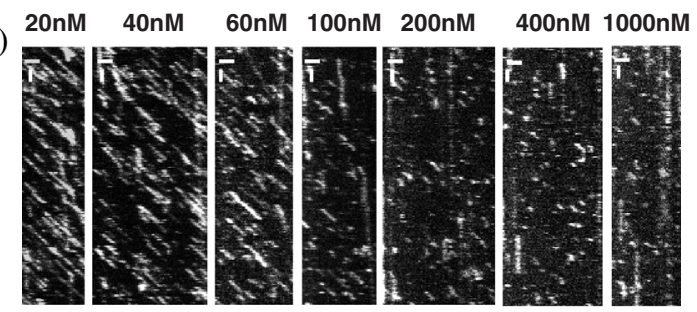

(b)

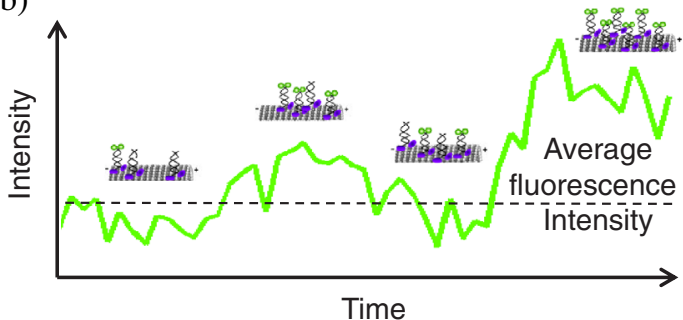

(c)

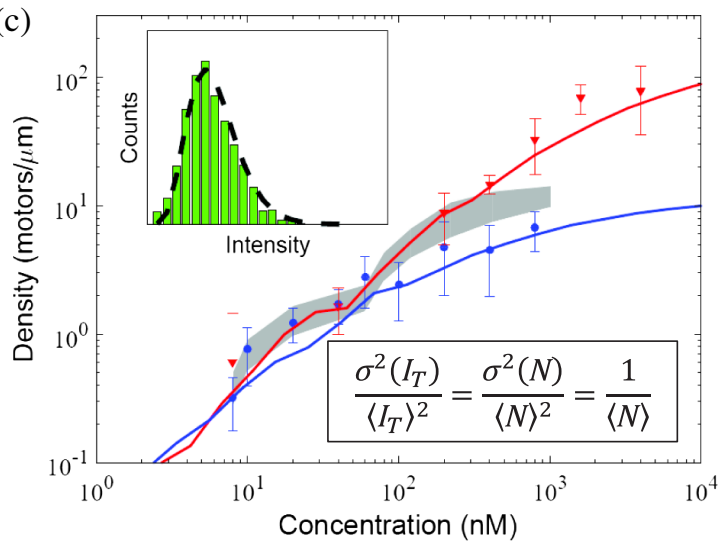

FIG. 3. Measurement of motor protein density from intensity fluctuations. (a) Kymographs [time-space plots; scale bars: $1 \mu \mathrm{m}$ (horizontal) and $1 \mathrm{~s}$ (vertical)] of kinesin-1 motility assays. The total of labeled and unlabeled motor concentration is indicated. Fluorescently labeled kinesin- 1 concentration is $20 \mathrm{nM}$ in all experiments. (b) Sketch of intensity fluctuations over time by random attachment and detachment of motors. (c) Motor density (labeled + unlabeled) on microtubule as a function of motor concentration in solution calculated from the intensity fluctuations. The density is obtained per microtubule for kinesin-1 (blue dots, 117 microtubule segments) and OSM-3 (red triangles, 76 microtubule segments). Error bars are standard deviations calculated for each concentration. Solid curves of the corresponding color show predictions of the extended TASEP-LK model (see main text). Grey shading (mean \pm standard deviation) indicates the estimate of density from manual particle counting of kinesin1. Inset: The normalized intensity follows a Poisson distribution (dashed line). Motility assays are performed in a PEM80 buffer. Numerical values of the experimental data used for fitting in panel (c) are listed in Table 2 in Ref. [17].

microtubule as shown in Fig. 4. The velocity and run-length plateau at low density and decrease at higher density because of crowding effects, in qualitative agreement with TASEP model predictions [Fig. 1(c)]. The velocity decrease suggests that interactions between motors slow their motion down, while the reduction in run length indicates the
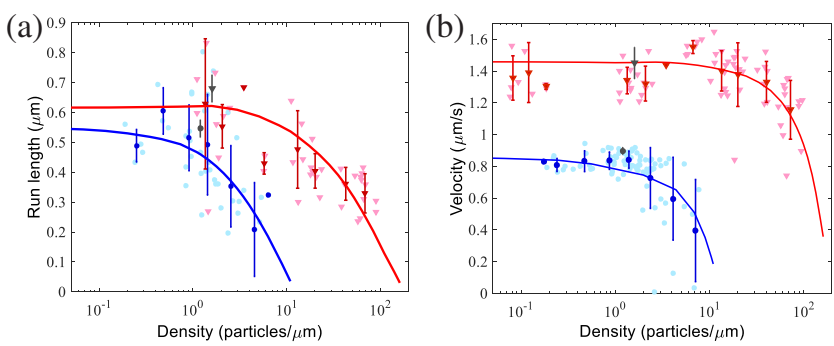

FIG. 4. Density-dependent run length and velocity: measurements and model predictions. (a) Run length as a function of density for kinesin-1 (blue dots) and OSM-3 (red triangles). Light dots show individual microtubule measurements, and dark symbols show averages and standard deviations over these measurements within density intervals of logarithmic size. Solid curves of the corresponding color show predictions of the extended TASEP-LK model (see main text). Single-particle measurements at low densities are shown in grey for comparison. (b) Velocity as a function of density. The plateau values of about $1.4 \mu \mathrm{m} / \mathrm{s}$ for OSM-3 and about $0.8 \mu \mathrm{m} / \mathrm{s}$ for kinesin- 1 are in good agreement with single-particle tracking measurements in dilute motor assays [18,21-23]. Motility assays are performed in a PEM80 buffer. Numerical values of the experimental data used for fitting are listed in Table 3 of Ref. [17].

competition for binding sites on the crowded microtubule. Similar to the density in Fig. 3(c), the decay occurs much earlier for kinesin-1 than for OSM-3. For OSM-3, the decay of velocity and run length starts at about 30 particles $/ \mu \mathrm{m}$, corresponding to an average motor separation of $16 \mathrm{~nm}$, indicating the motors start to noticeably interact when they fill half a single lane along the microtubule. At the same time, their maximum density of around 100 motors $/ \mu$ m indicates that the motors with size of $16 \mathrm{~nm}$ occupy more than one tubulin lane (protofilament). For kinesin-1, in contrast, the much lower crossover density of about 2 particles $/ \mu \mathrm{m}$ indicates that motors start to interact when the average distance is $0.5 \mu \mathrm{m}$, much larger than their physical size.

We note that the actual distances at which motors interact (Table 1 in Ref. [17]) are shorter than these average values, which rather indicate when the closest motors of the distribution start to interact. With increasing density, a larger number of motors come into the interaction range, resulting in the decreasing velocity and run length shown in Fig. 4. These interactions cause crowding of motors in the bulk of the microtubule. Indeed, we observe crowding everywhere along the microtubule, in contrast to a previous study, where crowding was observed at the microtubule ends only [8].

The different crowding behavior of the motors also persists when we change the buffer ionic strength. Using the lower-ionic-strength PEM12 buffer instead of PEM80, we observe that the onset of crowding occurs at the same density, and the velocity remains virtually unaffected. The run length, however, increases by a factor of 2-3 (Fig. 5 and Table 1 in Ref. [17]), indicating increased binding affinity of the motors to the microtubule. Interestingly, we also find, 
at lower ionic strength, that the correlation peak broadens more slowly with time, indicating that motor motion is smoother and less intermittent. We carefully examine the evolution of the correlation peak width with time and find that in the lower-salt PEM12 buffer, the variance of velocities increases much more slowly than in PEM80, and it does so consistently for both types of motors (Fig. 6 in Ref. [17]). Taken together with the constant average velocity, we conclude that the motor motion is less intermittent; i.e., motors change less prominently between a pausing and a walking state and thus spend less time pausing. This interpretation is indeed supported by highresolution kymographs that show a tendency towards straighter, less-intermittent, and longer single-molecule trajectories, as shown in Ref. [17], Figs. 6(e) and 6(f). This less intermittent motion is also reflected in the longer run length observed at lower salt concentrations: Pausing motors detach more easily from the microtubule and therefore lead to shorter run lengths, as discussed below.

\section{TESTING AND EXTENDING THE TASEP-LK MODEL}

To compare these measurements with theoretical predictions, we use the TASEP model with Langmuir kinetics (see Appendix C) to simulate the motion of motors along a filament. We use periodic boundary conditions in the simulations to investigate crowding in the bulk of the filament. The detachment rate $\omega_{D}$ is estimated from the low-density plateau of the velocity and run length as $\omega_{D}=v / \lambda$, which for OSM-3 yields $\omega_{D}=2.3 \mathrm{~s}^{-1}$; see Table 1 . The attachment rate $\omega_{A}$ is estimated from the initial linear growth of the filament density with motor concentration $c$ in solution. From the balance of motor attachment and detachment rates in the steady state, $\quad \rho \omega_{D} d=(1-\rho) \omega_{A}$, we determine that $\rho=$ $\left[\omega_{A} / d\left(\omega_{A}+\omega_{D}\right)\right]$, which reduces to $\omega_{A} \approx d \omega_{D} \rho$ in the dilute limit $\omega_{A} \ll \omega_{D}[9,10]$. Taking $\rho=1.64 \times 10^{-3} \mu \mathrm{m}^{2} \times c$ as determined from the slope in Fig. 3(c), the step size $d=0.008 \mu \mathrm{m}$, and $\omega_{D}=2.3 \mathrm{~s}^{-1}$ for OSM-3, we obtain $\omega_{A}=c \times 2.9 \times 10^{-5} \mu \mathrm{m}^{3} / \mathrm{s}$ (Table 1$)$.
Using these values of $\omega_{A}$ and $\omega_{D}$, we compute the densitydependent run length and velocity [dash-dotted black curves in Figs. 5(b) and 5(c)], which show poor quantitative agreement with the measurements. The reason for this is that in this simple TASEP-LK model, the velocity and run length always decrease together because of the constant binding time $\tau$ of the motors (Fig. 1), while in our measurements, the faster decaying run length indicates a density-dependent binding time. Furthermore, the very different interaction range observed for kinesin-1 and OSM-3 implies different maximum occupancy of the microtubule, which is different from single-lane occupancy assumed in the TASEP model.

To account for the different interaction range, we rescaled the density to the motor-specific crowding value: A crowding density lower than that of a fully occupied lane corresponds to motor interactions extended beyond simple geometric exclusion, while a higher density corresponds to motors walking in parallel along multiple lanes (without switching lanes); see Fig. 5(a). The best fit for OSM-3 is obtained with three parallel lanes, which very well reproduces the measured velocity [Fig. 5(c)], indicating that up to three motors can walk in parallel on a microtubule. This number is reasonable considering that in our in vitro experiments, about half of the lanes might be unavailable because of steric hindrance of the cover slip, leaving a maximum of five to six lanes available to the motors. In addition, motors might be hindered by motors on the next, parallel protofilament. We note that in vivo, however, more lanes on the microtubule may be available, and hence more motors may be able to walk in parallel.

While this extension of the TASEP-LK model describes the velocity reasonably well, the run length is still overestimated; see Fig. 5(b) (blue solid curve). Even if we allow the motors to switch lanes, which is reasonable as motors from the kinesin- 1 and kinesin- 2 families were observed to navigate past obstacles differently [24], this would still leave the data unexplained as it would rather increase the velocity than decrease the run length. Instead, the observed run-length decrease must result from enhanced detachment of constrained motors, which are abundant at high density.

TABLE I. Connecting TASEP-LK units to physical units for OSM-3 motor proteins in buffer solutions PEM80 on microtubules.

\begin{tabular}{|c|c|c|}
\hline Lattice unit size, nm & 8 & $\begin{array}{l}\text { The lattice unite size is equal to the length of one tubulin dimer in a } \\
\text { microtubule. }\end{array}$ \\
\hline Particle size, nm & 16 & A motor occupies two lattice sites (each motor head on one lattice site). \\
\hline Step size, nm & 8 & $\begin{array}{l}\text { The kinesin dimers walk in a head-over-head fashion, progressing half their } \\
\text { length in each step. }\end{array}$ \\
\hline Unit time, s & $5.7 \times 10^{-3}$ & $\begin{array}{l}\text { The kinesin dimers walk in a head-over-head fashion, progressing half the } \\
\text { particle size in each step. }\end{array}$ \\
\hline Detachment rate $\omega_{D}, \mathrm{~s}^{-1}$ & 2.3 & This is obtained from the run time of a free-moving motor. \\
\hline Attachment rate $\omega_{A}, \mathrm{~s}^{-1}$ & $\begin{array}{c}2.9 \times 10^{-5} \mu \mathrm{m}^{3} / \mathrm{s} \times \\
\text { concentration }\end{array}$ & $\begin{array}{l}\text { The concentration of motors in solution is an experimental control parameter. } \\
\text { The numerical constant is determined as the product of the detachment } \\
\text { rate, the lattice unit size, and the affinity [3-5], with the affinity measured } \\
\text { as the ratio of density and concentration in the low-density regime. }\end{array}$ \\
\hline
\end{tabular}



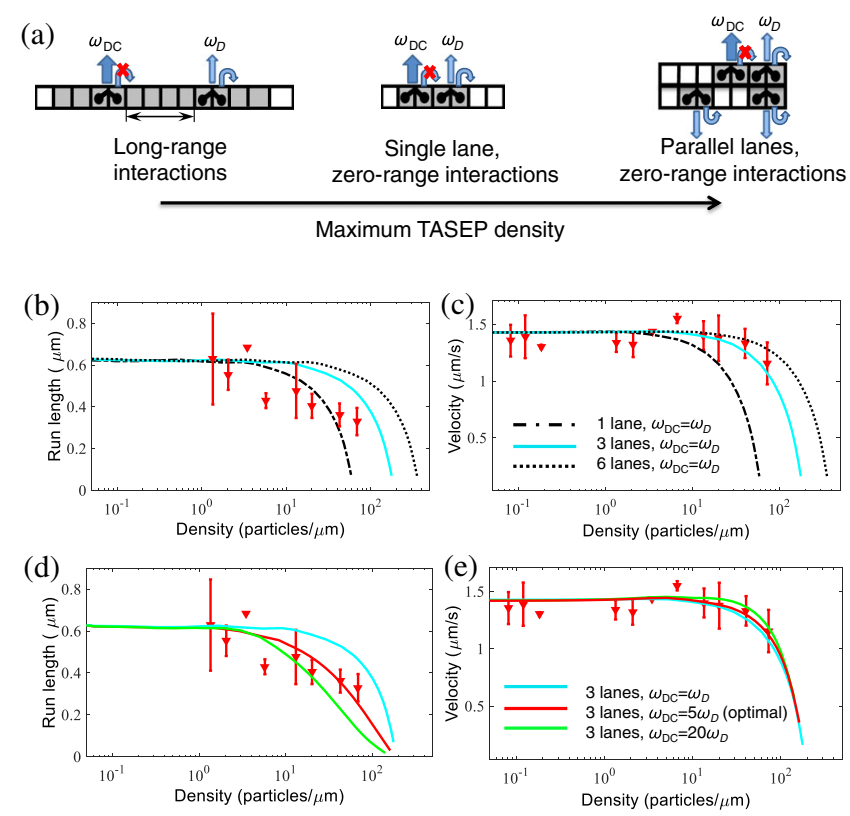

FIG. 5. Modeling of velocity and run length in the extended TASEP-LK model. (a) Schematic illustration of the density rescaling of the extended TASEP-LK model. Different motor interaction ranges and number of available lanes lead to different maximum density of the model. Three scenarios are shown: longrange interaction (low maximum density), short-range interaction of motors, single lane (medium maximum density), and shortrange interaction of motors, parallel lanes (high maximum density). Note that $\omega_{\mathrm{DC}}$ is the detachment rate of constrained motors, which differs from the detachment rate of unconstrained motors $\omega_{D}$ in the extended model; this is illustrated by arrows of different thickness. (b-e) TASEP-LK model predictions of run length and velocity for OSM-3 motor proteins performed in a PEM80 buffer. (b,c) Variation of the number of parallel lanes. The best fit of the experimental data (red triangles and error bars) is obtained with three parallel lanes. (d,e) Variation of the detachment rate $\omega_{\mathrm{DC}}$ of constrained motors for three parallel lanes. The best fit for the run length is obtained with $\omega_{\mathrm{DC}}=11.5 \mathrm{~s}^{-1}$ (solid red curve), while the velocity is only weakly affected by $\omega_{\mathrm{DC}}$.

We hypothesize that unlike the original TASEP-LK assumption of a fixed detachment rate, constrained motors detach from the filament with an enhanced rate. This seems reasonable considering that a constrained motor likely has only one of its motor domains bound to the microtubule and is thus expected to detach more easily than a walking motor. Previous experiments on kinesin motors crowded by static microtubule binding proteins support this idea [18]. We hence introduce a second detachment rate $\omega_{\mathrm{DC}}$ for constrained motors, as illustrated in Fig. 5(a). Using this additional parameter, we obtain excellent fits of the data, as shown by the red solid curves in Figs. 5(d) and 5(e), using $\omega_{\mathrm{DC}} \sim 11.5 \mathrm{~s}^{-1}$, which is 5 times higher than the detachment rate of unconstrained motors $\omega_{D} \sim 2.3 \mathrm{~s}^{-1}$. Because a motor takes around 175 steps per second, as determined from its average free velocity and step size of $8 \mathrm{~nm}$, this implies that for each step, constrained OSM-3 motors detach from the microtubule with probability 0.06 or stay attached and pause with probability 0.94 , while unconstrained motors detach with probability 0.01 or step with probability 0.99 . These best fits of the OSM-3 data are indicated in red in Figs. 3(c) and 4.

For kinesin-1, the crowding occurs at much lower densities because of the much longer interaction range of the motors. We find that using an interaction range as long as about $60 \mathrm{~nm}$, and a detachment probability of constrained motors of 0.05 , we are able to fit the experimental data well [blue curves in Fig. 3(c), Fig. 4, as well as Fig. 7 in Ref. [17]]. The longer interaction range leads to an effective occupancy of less than a single lane as assumed by standard TASEP, which is also very different from the multilane scenario of OSM-3. These results highlight the very different way these motors deal with crowding effects.

Long-range interactions of kinesin-1 have indeed been observed before [25], together with increased attachment rates of motors in the presence of already-attached motors. It is unlikely that electrostatic interactions between motor proteins play a role over distances of $0.1-1$ micrometer at the ionic conditions used in our experiments since the corresponding Debye screening length is about a nanometer. Elastic distortion of the protofilaments could explain binding of kinesin-1 at long ranges [26]; however, this model fails to capture the enhanced detachment under crowding at ranges of about 1 micrometer that we observe here. Hence, the physical mechanism underlying these long-range interactions still needs to be clarified.

\section{DISCUSSION AND CONCLUSIONS}

Our first combined, quantitative measurements of motor density, velocity, and run length on highly crowded filaments allow the quantitative assessment of nonequilibrium models of molecular motor transport from experimental data. These measurements confirm crowding effects in quantitative agreement with an extended TASEP-LK model, validating the applicability of this basic nonequilibrium model in the biological molecular motor transport. Previous experiments on static crowders such as Tau [27] and kinesin rigor mutants [18] already showed the negative effect of crowding on both velocity and run length of kinesin-1. Heterotrimeric kinesin-2 motors from mouse were shown to be less affected by Tau crowding, likely because of the motors' ability to sidestep the blocked microtubule sites [24]. Dynamic crowding of kinesin-1 by moving motors revealed similar crowding effects $[19,28,29]$, clearly showing that crowding impacts the motor motility, but a quantitative assessment in the context of physical transport models remained elusive.

Our measurements of dense kinesin-1 and OSM-3 motor transport not only confirm basic model predictions but also reveal motor-specific strategies to deal with crowding, which are well in line with the general characteristics of 
the motors. Kinesin-1 is a motor protein that is thought to transport cargo on its own or, at most, in small groups [30,31]. In vitro assays of coupled kinesin-1 motors showed that only one of the motors is effectively engaged at a time [32]. On the other hand, OSM-3, a homodimeric member of the kinesin-2 family from $C$. elegans involved in intraflagellar transport [33], moves in teams of about 40 OSM-3 motors to cargo trains, consisting of many hundreds of proteins, along the microtubules in cilia [34]. In addition, the OSM-3-driven motility of such trains is in a regulated competition with kinesin-II, a heterotrimeric kinesin-2 motor walking at a slower pace in the same direction, up to 60 of which occupy a single train. It is therefore not surprising that OSM-3's motility properties are better optimized for teams working at closer distances than those of kinesin-1.

While our measurements give an accurate and reproducible picture of crowded motor transport in in vitro assays, it will be intriguing to see how these properties carry over to the in vivo cell environment, as well as to coupled motors. For example, the attachment of cargo or the entrainment of motors can greatly alter their motile and detachment properties. Also, additional crowding agents in the cell environment can greatly change the motors' dynamic behavior, clearly showing the need for additional in vivo experiments. It may be expected that entrained motors and those pulling on the same cargo, or even those in a crowded cell environment, exhibit less detachment and longer persistence length on the microtubule, leading to even stronger jamming effects. On the other hand, loose random crowding agents in the cell can also lead to decorrelation, preventing jam formation. While this remains speculative, our in vitro assays clearly reveal motor-specific crowding effects in both their velocity and run length. The combination of TASEP modeling and microscopic correlation imaging hence provides fundamental insight into the interactions of individual motors at the nano scale.

\section{ACKNOWLEDGMENTS}

This work was financially supported by the Complexity Initiative of the Netherlands Organization for Scientific Research (NWO) (D. M. M, D. V. D., B. N., and P. S.), by NWO VICI grants (P. S. and E. P.), and by the programme grant "Barriers in the Brain" from the Foundation for Fundamental Research on Matter (FOM) (E. P.)

D. M. M. and V. S. K. contributed equally to this work.

\section{APPENDIX A: EXPERIMENTAL METHODS}

All chemicals were purchased from Sigma-Aldrich, unless stated differently. All concentrations given are final concentrations.

Microtubules preparation.-Microtubules (MTs) were prepared from in-house purified tubulin from bovine brain tubulin as described previously [35]. Tubulin $(5 \mathrm{mg} / \mathrm{ml})$ in PEM80 [80 mM PIPES pH 6.9, 1 mM EGTA, 2 mM MgCl 2 (Fluka analytical 63072)] buffer supplemented with $4 \mathrm{mM}$ $\mathrm{MgCl}_{2}, 1 \mathrm{mM}$ GMPCPP (nonhydrolyzable GTP-analogue, Jena Bioscience NU-405S,), and 5\% DMSO was incubated for an hour at $37^{\circ} \mathrm{C}$ to polymerize. The microtubules were stabilized by mixing 1:20 (v/v) with PEM 80 containing $10 \mu \mathrm{M}$ taxol [36].

Motor proteins.-The two motor proteins used in the experiments were Drosophila melanogaster homodimeric kinesin-1 (conventional kinesin) and Caenorhabditis elegans homodimeric OSM-3. These motors are processive motors, and they walk towards the plus end of MTs.

Kinesin-1.-The DNA sequence coding for the first 432 amino acids of conventional kinesin from Drosophila melanogaster was amplified with the primers by polymerase chain reaction and cloned into pETDuet-1 vector (Addgene) generating pETDuet_1MCS1_dmKin 432 . As a fluorescent tag, the gene of monomeric superfolded (sfGFP), and the Strep-tagII sequence as a purification tag were introduced to the C-terminal end of the kinesin sequence by including the sequence in the primer used for cloning, resulting in the pETDuet1_MCS1_dmKin ${ }_{432}$ sfGFP_StreptagII plasmid. This plasmid was transformed into Rosetta-gami ${ }^{\mathrm{TM}}$ 2(DE3) pLysS competent cells (Novagen). Protein expression was induced by addition of $0.4 \mathrm{mM}$ IPTG to a $1.6 \mathrm{~L}$ culture in the Erlenmeyer flasks. Expression was allowed to proceed for 6 hours at $22^{\circ} \mathrm{C}, 220 \mathrm{rpm}$ in a shaker incubator (Infors HT-Ecotron). Expressed proteins were purified from the lysate using Strep-Tactin affinity chromatography (IBA Life Sciences), like the other Strep-tagged proteins below. The plasmid of unlabeled kinesin-1 motor proteins, pETDuet1_MCS1_dmKin ${ }_{432}$ SNAP_His, was transformed, expressed, and purified using Ni-NTA affinity chromatography (QIAGEN), like the other His-tagged proteins below. The concentration of purified kinesin1_sfGFP and unlabeled kinesin-1 proteins were $1.8 \mathrm{mg} / \mathrm{ml}$ and $2.5 \mathrm{mg} / \mathrm{ml}$, respectively, as measured using Bradford assays with bovine serum albumin as standard [37]. Freshly purified motor proteins in PEM80 buffer containing $20 \%(\mathrm{v} / \mathrm{v})$ sucrose were aliquoted in $10 \mu \mathrm{l}$ aliquot, snap frozen in liquid nitrogen, and stored at $-80^{\circ} \mathrm{C}$ until use.

OSM-3.-Similarly, using fusion PCR strategy, the DNA sequence coding for the motor and neck linker of Caenorhabditis elegans OSM-3 (341aa), the minimal part of kinesin-1 stalk for dimerization (88aa), and sfGFP coding sequence were amplified with the primers by polymerase chain reaction and cloned into pETDuet-1 vector (Addgene) generating pETDuet1_MCS1_ceOsm3_6XHis. The plasmid of unlabeled OSM-3 motor proteins, pETDuet1_MCS1_ceOsm-3_SNAP_His, contained a Snap tag instead of a fluorescent tag. All of the constructs were transformed, expressed, and purified as explained 
for kinesin-1 above. Freshly purified motor proteins in PEM80 buffer containing 20\% (v/v) sucrose were aliquoted in $10 \mu \mathrm{l}$ aliquot, snap frozen in liquid nitrogen, and stored at $-80^{\circ} \mathrm{C}$ until use. The protein concentrations for the purified OSM-3_sfGFP and unlabeled OSM-3 were $1.3 \mathrm{mg} / \mathrm{ml}$ and $2.9 \mathrm{mg} / \mathrm{ml}$, respectively. Protein concentrations were determined using Bradford assays with bovine serum albumin as standard [37].

Glass surface preparation.-Hydrophobic surfaces were used for the motility assays in order to strongly adsorb antibodies against tubulin to the glass surface and to allow efficient surface blocking using amphiphilic pluronic F127. Glass cleaning: Glass coverslips and microscope slides were first plasma cleaned for around $20 \mathrm{~min}$ and incubated in $0.1 \mathrm{M} \mathrm{KOH}$ for $15 \mathrm{~min}$. After another 1-min and 2-min wash sequence with Milli-Q water, the coverslips and slides were blown dry with argon gas. Silanization: $100 \mu \mathrm{l}$ DDS (dichlorodimethylsilane) was dissolved in $200 \mathrm{ml}$ TCE (trichloroethylene) in a glass beaker. Cleaned coverslips were incubated in this solution for $60 \mathrm{~min}$ at room temperature, followed by two steps of sonication for $5 \mathrm{~min}$ and $15 \mathrm{~min}$, both in methanol at room temperature. Finally, cleaned and hydrophobic slides and coverslips were blown dry using argon gas and transferred into clean holders and stored in a closed glass box, sealed with parafilm [36]. Flow-cell assembly: Assays were performed in hydrophobic flow cells made of microscope glass slides (Menzel-Glaser, $76 \times 26 \mathrm{~mm}$, cut edges) attached to a coverslip (High Precision, Deckglaser, $22 \times 22 \mathrm{~mm}$, thickness $170 \pm 5 \mu \mathrm{m}$ ) with double-sided Scotch tape, resulting in flow channels of about $170 \mu \mathrm{m}$ inner height with a volume of approximately 5-8 $\mu \mathrm{l}$.

Instrumentation.-Assays were performed at $23^{\circ} \mathrm{C}$ using Total Internal Reflection Fluorescence (TIRF) microscopy. Microscope images were acquired using a custom-built TIRF microscope operated by the Micro-Manager software interface (Micro-Manager 1.4), built around an inverted microscope body (Nikon, Eclipse Ti) equipped with a 100x oil-immersion objective (Nikon, CFI Apo TIRF 100X, numerical aperture 1.49) and with a motorized actuator (ThorLabs Z825B) along with a control cube (TDC001). The excitation light, provided by a diode-pumped solidstate laser (Cobolt Calypso ${ }^{\mathrm{TM}}$ 50-mW 491-nm DPSS), first passed through an AOTF (AA Opto-Electronics, AOTFnC-400.650-TN) for wavelength selection, then through a quarter wave-plate (Thorlabs, mounted achromatic quarter-wave plate, 400-800 nm, AQWP05M-600) to obtain circularly polarized light, and finally through a dichroic mirror (Semrock, 405/488/561/635-nm lasers Brightline@quad-edge laser-flat, Di 01-R405/488/561/ $635-25 \times 36$ ). The intensity of the $491-\mathrm{nm}$ laser was estimated to be about $81 \mathrm{~W} / \mathrm{cm}^{2}$ in the image plane. The emitted fluorescent light was separated inside the Optosplit III using a dichroic long-pass filter. Images were acquired continuously by an EMCCD camera (Andor, iXon 897 ) without delay in between and saved as 16-bit tiff files. One camera pixel corresponds to $80 \mathrm{~nm} \times 80 \mathrm{~nm}$ in the image plane. Special care was taken to minimize the effect of photobleaching and possible potential sources of noise while performing the experiments without compromising temporal resolution and SNR.

Kinesin-1-MT crowding assays.-To immobilize the microtubules, the sample chambers were first incubated with a $2-\mu \mathrm{g} / \mathrm{ml}$ monoclonal anti- $\beta$-tubulin antibody in a PEM80 buffer with $10 \mu \mathrm{M}$ taxol (hereafter denoted as PEM80T). After 5 min of incubation, the excess antibodies were flushed out with PEM80T, followed by $15 \mathrm{~min}$ of incubation with $1 \%(\mathrm{w} / \mathrm{v}$ ) Pluronic F-127 in PEM80T. To flush out the excess Pluronic F127, the chambers were rinsed with PEM80T after 15 min of incubation. In the next step, the sample chambers were incubated with $0.3-\mathrm{mg} / \mathrm{ml}$ microtubules in PEM80T, allowing the microtubules to attach to the surface via the antibodies for $10 \mathrm{~min}$. After attaching the microtubules, the sample chambers were flushed with PEM80T (or PEM12T, to perform motility assays in lower ionic buffer conditions, a PEM80 buffer was replaced with a PEM12 buffer in another set of experiments). In the final step, the sample chambers were flushed with motility solution in PEM80T (or PEM12T) supplemented with $2 \mathrm{mM} \mathrm{MgCl} 2,2 \mathrm{mM}$ ATP (Adenosine 5'-Triphosphate), $0.2 \mathrm{mg} / \mathrm{ml}$ casein in PEM80, an ATP regeneration system (Phosphocreatine $10 \mathrm{mM}$, Creatine phosphokinase $0.05 \mathrm{mg} / \mathrm{ml}$ ), an oxygen scavenging cocktail containing $216 \mu \mathrm{g} / \mathrm{ml}$ glucose oxidase, $36 \mu \mathrm{g} / \mathrm{ml}$ catalase, $22.5 \mathrm{mM}$ glucose, and $10 \mathrm{mM}$ of DTT (dithiothreitol), and kinesin- 1 motor proteins. We varied the final concentration of kinesin-1 motor proteins from $20 \mathrm{nM}$ to $1000 \mathrm{nM}$ in the motility mix by diluting sfGFP-labeled motors with unlabeled motors at motor ratios of $1: 0,1: 2$, $1: 3,1: 5,1: 10,1: 20$, and $1: 50$, while the concentration of labeled motor proteins was kept constant at $20 \mathrm{nM}$. The sample chambers were sealed with VaLaP (equal parts vaseline, lanoline, and paraffin). The kinesin-1_sfGFP motors were illuminated by the 491-nm laser, and moving motors were observed under the TIRF microscope setup. One thousand images were acquired continuously with a $100-\mathrm{ms}$ exposure time without delay in between. Multiple microtubules (2-5) were analyzed per image stack, adding up to a total of 117 analyzed microtubules for kinesin-1 in PEM80 buffer and 53 analyzed microtubules in PEM12 buffer.

OSM-3-MT crowding assays. - In these assays, after the microtubule immobilization step, the sample chambers were flushed with motility solution in PEM80T (or PEM12T) supplemented with OSM-3 motor proteins, $2 \mathrm{mM} \mathrm{MgCl} 2$, $2 \mathrm{mM}$ ATP (Adenosine 5'-Triphosphate), $0.2 \mathrm{mg} / \mathrm{ml}$ casein in PEM80, an ATP regeneration system (Phosphocreatine $10 \mathrm{mM}$, Creatine phosphokinase $0.05 \mathrm{mg} / \mathrm{ml}$ ), an oxygen scavenging cocktail containing $216 \mu \mathrm{g} / \mathrm{ml}$ glucose oxidase, 
$36 \mu \mathrm{g} / \mathrm{ml}$ catalase, $22.5 \mathrm{mM}$ glucose, and $10 \mathrm{mM}$ of DTT (dithiothreitol). The estimated final concentration of OSM-3 was varied from $40 \mathrm{nM}$ to $4000 \mathrm{nM}$ by diluting the OSM-3_sfGFP labeled with unlabeled OSM-3 motors at motor ratios of $0.2: 0,1: 0,1: 10,1: 20,1: 40$, and $1: 100$, while the concentration of labeled motor proteins was kept constant at $40 \mathrm{nM}$. Finally, the sample chambers were sealed with $\mathrm{VaLaP}$ (equal parts vaseline, lanoline, and paraffin). The OSM-3_sfGFP motor proteins were excited with the 491-nm laser. Moving OSM-3 motor proteins were observed under a microscope setup. One thousand images were acquired continuously with either a 50-ms or 70-ms exposure time without delay in between. Multiple microtubules (2-5) were analyzed per image stack, adding up to a total of 76 analyzed microtubules for OSM-3 in PEM80 buffer and 69 analyzed microtubules in PEM12 buffer.

\section{APPENDIX B: CORRELATION IMAGING}

Correlation of intensities. - We manually chose the beginning and end point of the microtubule segment to be analyzed and align the microtubule axis along the $x$ direction of our coordinate system. At each position $x$ along the microtubule axis, the time-dependent intensity $I(x, t)$ was measured with pixel resolution over a width of $w=80 \mathrm{~nm}$. The background intensity measured in the direct vicinity of the microtubule was subtracted. Timeaveraged intensities were typically constant along microtubules; when significant heterogeneities were observed, e.g., due to crossing microtubules, these regions were excluded from the analysis. The normalized correlation of intensities $C$ was calculated over temporal separation ( $d t$, minimum $50 \mathrm{~ms}$ for OSM-3 and $100 \mathrm{~ms}$ for kinesin-1) and spatial separation ( $d x$, minimum $80 \mathrm{~nm}$ ) using

$$
C_{0}(d t, d x)=\frac{\langle(I(x, t)-\langle I(x, t)\rangle)(I(x+d x, t+d t)-\langle I(x+d x, t+d t)\rangle)\rangle}{\sigma(I(x, t)) \sigma(I(x+d x, t+d t))},
$$

with

$$
\sigma(I(x, t))=\sqrt{\left\langle(I(x, t)-\langle I(x, t)\rangle)^{2}\right\rangle}
$$

being the standard deviation of the intensity distribution. The averages in Eq. (B1), denoted by the triangular brackets, are taken over both space and time.

Correction for noise in intensities. - Besides the signal of the functional, fluorescently labeled motors, various sources contribute to the background-corrected intensities on the microtubules: (1) fluorescently labeled, nonfunctional motors, (2) inhomogeneities in illumination, and (3) random noise. By definition, the random noise does not affect the calculation of velocity and run length as it only contributes a random component to $I(x, t)$ and can therefore easily be shown to average out to zero in the numerator of Eq. (B1) for al $d t>0$. Random noise does contribute to the standard deviation of the intensities in the denominator of Eqs. (B1) and (B2), but as there is no $d x$ or $d t$ dependence in the standard deviation, this does not affect the calculation of velocity or run length, which are both derived from the correlation peak moving at $d x=v d t$.

Nonfunctional fluorescently labeled motors and inhomogeneities in illumination both contribute static noise to the correlation profile. In contrast, the functional motors are dynamic, such that we can calculate the long-term (much longer than the run times of motors $d t \gg 1 / \omega_{D}$, e.g., more than 2 seconds) correlation profile and subtract this from the correlation at all times to remove the influence of the static noise and arrive at a correlation $C$ from which functional motor properties can be derived,

$$
C(d t, d x)=C_{0}(d t, d x)-\left\langle C_{0}(d t, d x)\right\rangle_{d t \gg 1 / \omega_{D}} .
$$

We use this background-corrected correlation function [Eq. (B3)] of intensity fluctuations for the determination of motor velocities and run lengths to exclude any bias or artefacts from static noise sources.

Velocity.-Fluorescently labeled motors introduce peaks in the intensity profile, while the absence of fluorescently labeled motors introduces valleys in the intensity profile. Motor movement with velocity $v$ over a time interval $d t$ causes a spatial translation $d x=v d t$ of this intensity profile, resulting in a positive correlation along a line in space-time [Fig. 2(c), as well as Fig. 3(b) in Ref. [17]]. As a result, the cross-correlation of intensities [Eq. (B3)] is maximal at $d x=v d t$, and the average velocity can be derived from tracking of the correlation peak [14].

Variance of velocity. - The width of the correlation peak is quantified by the variance obtained from Gaussian fitting. The initial variance is set by the diffractionlimited size of fluorescent motors and the finite observation time. If all motors travel a distance $d x=v d t$ during a time interval $d t$, the width of the correlation peak remains constant. If, however, there is a distribution of distances traveled, the correlation peak after a time $d t$ is a convolution of the distribution of distances traveled and the peak from a single fluorescent motor. Hence, the variance of velocities at time $d t$ can be derived from the width of the correlation peak at $d t$ minus the variance of the initial width (which is identical to that of a single fluorescent motor); see Fig. 6 in Ref. [17]. 
Run length.-Fluctuations in motor velocity result in a decrease of the height of the correlation peak and an increase in the width of the correlation peak, but they leave the area under the correlation peak $A$ unchanged [15]. On the other hand, attachment and detachment result in a decrease of the correlation of intensities and hence a decrease in $A$. For convenience, we ignore fluctuations in the velocities (as this does not change $A$ ) and use the decrease of the correlation peak height $[C(d t, d x=v d t)]$ with $d t$ to derive the run length. The height of the peak $C(d t, d x=v d t)$ is proportional to $A(d t)$ because the width of the peak remains constant if all motors have a constant velocity.

The correlation is calculated from the deviation of the average intensity measured in cross sections along the length of a microtubule segment at time $t$ and $t+d t$. The intensity in a cross section containing $n$ fluorescently labeled motors is $I_{m} n$, if we assume that all fluorescent motors have equal intensity $I_{m}$ and ignore noise (the contribution of noise to the correlation of intensities is independent of $d t$ and $d x$ ), while the intensity in the absence of a motor is 0 . The density of fluorescently labeled motors $\rho_{L}$ determines the fraction of cross sections $f=\rho_{L} \cdot w$ that are occupied by fluorescently labeled motors and the average intensity $\langle I\rangle=f I_{m}$. The density of fluorescent motors in our experiments is about 1 motor/ micrometer (the total motor concentration was much higher in some experiments, but this was reached by adding motors without a fluorescent label), from which $f \sim 0.1$ can be derived. Thus, the majority of cross sections contain no motor [fraction $1-f$, intensity $I(x, t)=0$ ] or a single motor $n=1$ [fraction $f, I(x, t)=I_{m}$ ]; the probability that two motors are in the same cross section is of the order $f^{2} \sim 0.01$. Before any attachment or detachment occurred $[I(t, x)=I(t+d t, x+d x)]$, the correlation can thus be approximated as

$$
\begin{aligned}
& C(d t, d x=v d t)^{\mathrm{nodet} / \mathrm{att}} \\
& \quad \sim f\left(I_{m}-f I_{m}\right)^{2}+(1-f)\left(0-f I_{m}\right)^{2},
\end{aligned}
$$

where the first term in Eq. (B4) represents the contribution of cross sections containing fluorescently labeled motors, while the second term contains the contribution from cross sections not containing a fluorescent motor. The relative contribution of fluorescently labeled motors to the correlation can be estimated from the ratio of the first term to the total:

$$
\frac{f\left(I_{m}-f I_{m}\right)^{2}}{f\left(I_{m}-f I_{m}\right)^{2}+(1-f)\left(0-f I_{m}\right)^{2}}=1-f,
$$

which is close to 1 for the values of $f \sim 0.1$ in our experiments, so we can neglect the second term in Eq. (B4). The correlation in the absence of attachment or detachment, Eq. (B4), can thus be approximated by the correlation deriving from cross sections containing a fluorescently labeled motor:

$$
C(d t, d x=v d t)^{\text {no det/att }} \sim f\left(I_{m}-f I_{m}\right)^{2} .
$$

The correlation is lost over time by the attachment and/or detachment of fluorescently labeled motors:

$C(d t, d x=v d t) \sim f\left(I_{m}-f I_{m}\right)^{2}\left(1-p_{D}\right)\left(1-p_{A}\right)$.

Here, we introduce the probabilities $p_{A}$ and $p_{D}$ of motor attachment and detachment in a cross section containing a fluorescent motor, respectively, during time interval $d t$ at position $v d t$. Summed over the whole microtubule, the detachment and attachment of fluorescently labeled motors balances in the steady state because of the conservation of the number of motors. Because detachment only occurs in cross sections containing a fluorescently labeled motor and attachment can occur in all cross sections, we find $p_{D}=p_{A} / f$. The decrease in correlation from the detachment is thus much larger (factor $1 / f>10$ ) than the decrease resulting from the attachment, and over the entire range of concentration that we use in our experiments with $p_{A} \ll 1$, we can thus neglect the term $1-p_{A} \approx 1$ so that

$C(d t, d x=v d t) \sim f\left(I_{m}-f I_{m}\right)\left(I_{m}-f I_{m}\right)\left(1-p_{D}\right)$.

Using Eq. (B6) to replace the first terms in Eq. (B8), we thus obtain the following expression for the loss of correlation due to motor detachment in terms of $p_{D}$ :

$$
\frac{C(d t, d x=v d t)}{C(d t, d x=v d t)^{\text {no det } / \mathrm{att}}}=1-p_{D}(d t),
$$

where the right-hand side simply represents the probability that a fluorescent motor is still attached after a time $d t$. If the stepping and detachment of motors is a random (Poisson) process, this probability decays exponentially with time:

$$
1-p_{D}(d t)=e^{-\omega_{D} d t},
$$

where $\omega_{D}$ is the detachment rate of motors from the microtubule. (Note that $\omega_{D}$ and $\omega_{A}$ are the detachment and attachment rates per motor, while the above-introduced $p_{D}$ and $p_{A}$ are the detachment and attachment rates per cross section containing a fluorescent motor.) Indeed, we find experimentally that the area $A(d t)$ under the correlation curve [which is proportional to $C(d t, d x=v d t)$ ] decays exponentially [Fig. 2(f), as well as Fig. 3(e) in Ref. [17]]. We then obtain $\omega_{D}$ by fitting a simple exponential decay function [right-hand side of Eq. (B10)] to $A(d t)$ and finally calculate the run length as $\lambda=v / \omega_{D}$.

As some approximations were made in the above calculation of the run length, we validated the correlation imaging technique by comparing the resulting values for 
velocity and run length with results from single-particle tracking (Fig. 2 in Ref. [17]) and literature and find excellent agreement. In particular, we confirmed that correlation imaging is applicable over the whole range of densities of fluorescent motors used in our experiments [Fig. 2(g) in Ref. [17]].

Motor density.-The motor density on a microtubule segment was calculated by fluorescent correlation spectroscopy from the temporal intensity fluctuations (spatially averaged) as described in the main text.

Code availability.-Custom-written routines in MATLAB (MathWorks, Natick, MA) were developed to automate the image analysis of correlation imaging. The complete analysis code to extract motility parameters from image sequences using correlation imaging is available at [38].

\section{APPENDIX C: PARTICLE TRACKING AND TASEP}

Single particle tracking.-Fluorescence image sequences were analyzed using custom-written routines in MATLAB (MathWorks, Natick, MA), based on the singleparticle tracking algorithm utrack [39]. Trajectories with a total length of more than four subsequent frames were taken into account for velocity and run-length determination. Kymographs (time-space plots) were generated using a custom-written ImageJ plugin [40].

TASEP-LK model.-The totally asymmetric simple exclusion process model is a simple stochastic microscopic model for systems in one dimension with hard-core particle interactions [3-5]. Particles progress unidirectionally with steps of size 1 along a segment of length $L=1000$ if the target site is empty, and they do not move otherwise. Periodic boundary conditions were applied to study bulk crowding effects. Particles were taken to occupy two lattice sites to mimic the two-head binding of kinesin motors. Particle dynamics are updated in random sequential order for $10^{5}$ update cycles (each cycle corresponds to $L$ events). To include binding and unbinding of particles, the TASEP model was extended with Langmuir kinetics (LK). With LK, a bound particle unbinds with rate $\omega_{D}$, while an empty lattice site becomes populated with a newly binding particle with rate $\omega_{A}$ each time step. We introduced an enhanced detachment rate $\omega_{D C}$ for constrained motors to explain the faster decay of run length with density compared to decay of velocity with density.

[1] J. Howard, Mechanics of Motor Proteins and the Cytoskeleton (Sinauer Associates, Sunderland, Massachusetts, 2001).

[2] E. L.F. Holzbaur and Y.E. Goldman, Coordination of Molecular Motors: From In Vitro Assays to Intracellular Dynamics, Curr. Opin. Cell Biol. 22, 4 (2010).
[3] C. T. MacDonald, J. H. Gibbs, and A. C. Pipkin, Kinetics of Biopolymerization on Nucleic Acid Templates, Biopolymers 6, 1 (1968).

[4] C. T. MacDonald and J. H. Gibbs, Concerning the Kinetics of Polypeptide Synthesis on Polyribosomes, Biopolymers 7, 707 (1969).

[5] A. Parmeggiani, T. Franosch, and E. Frey, Phase Coexistence in Driven One-Dimensional Transport, Phys. Rev. Lett. 90, 086601 (2003).

[6] K. Nishinari, Y. Okada, A. Schadschneider, and D. Chowdhury, Intracellular Transport of Single-Headed Molecular Motors KIF1A, Phys. Rev. Lett. 95, 118101 (2005).

[7] K. Tsekouras and A. B. Kolomeisky, Parallel Coupling of Symmetric and Asymmetric Exclusion Processes, J. Phys. A 41, 465001 (2008).

[8] C. Leduc, K. Padberg-Gehle, V. Varga, D. Helbing, S. Dietz, and J. Howard, Molecular Crowding Creates Traffic Jams of Kinesin Motors on Microtubules, Proc. Natl. Acad. Sci. U.S.A. 109, 6100 (2012).

[9] A. Parmeggiani, T. Franosch, and E. Frey, Totally Asymmetric Simple Exclusion Process with Langmuir Kinetics, Phys. Rev. E 70, 046101 (2004).

[10] I. Neri, N. Kern, and A. Parmeggiani, Modeling Cytoskeletal Traffic: An Interplay between Passive Diffusion and Active Transport, Phys. Rev. Lett. 110, 098102 (2013).

[11] R. D. Vale, T. S. Reese, and M. P. Sheetz, Identification of a Novel Force-Generating Protein, Kinesin, Involved in Microtubule-Based Motility, Cell 42, 39 (1985).

[12] A. Yildiz, M. Tomishige, R. D. Vale, and P. R. Selvin, Kinesin Walks Hand-over-Hand, Science 303, 676 (2004).

[13] L. Cipelletti, G. Brambilla, S. Maccarron, and S. Caroff, Simultaneous Measurement of the Microscopic Dynamics and the Mesoscopic Displacement Field in Soft Systems by Speckle Imaging, Opt. Express 21, 22353 (2013).

[14] S. Buzzaccaro, E. Secchi, and R. Piazza, Ghost Particle Velocimetry: Accurate 3D Flow Visualization Using Standard Lab Equipment, Phys. Rev. Lett. 111, 048101 (2013).

[15] M. G. Olsen and R.J. Adrian, Brownian Motion and Correlation in Particle Image Velocimetry, Opt. Laser Technol. 32, 621 (2000).

[16] L. Ciandrini, I. Neri, J. C. Walter, O. Dauloudet, and A. Parmeggiani, Motor Protein Traffic Regulation by SupplyDemand Balance of Resources, Phys. Biol. 11, 056006 (2014).

[17] See Supplemental Material at http://link.aps.org/ supplemental/10.1103/PhysRevX.7.041037 for additional data and figures on the experiments, analysis and modeling.

[18] I. A. Telley, P. Bieling, and T. Surrey, Obstacles on the Microtubule Reduce the Processivity of Kinesin-1 in a Minimal In Vitro System and in Cell Extract, Biophys. J. 96, 3341 (2009).

[19] L. Conway, D. Wood, E. Tüzel, and J. L. Ross, Motor Transport of Self-Assembled Cargos in Crowded Environments, Proc. Natl. Acad. Sci. U.S.A. 109, 20814 (2012).

[20] E. L. Elson, Fluorescence Correlation Spectroscopy: Past, Present, Future, Biophys. J. 101, 2855 (2011).

[21] M. Imanishi, N. F. Endres, A. Gennerich, and R. D. Vale, Autoinhibition Regulates the Motility of the C. elegans Intraflagellar Transport Motor OSM-3, J. Cell Biol. 174, 931 (2006). 
[22] X. Pan, G. Ou, G. Civelekoglu-Sholey, O. E. Blacque, N. F. Endres, L. Tao, A. Moligner, M. R. Leroux, R. D. Vale, and J. M. Scholey, Mechanism of transport of IFT particles in C. elegans cilia by the concerted action of kinesin-II and OSM-3 motors, J. Cell Biol. 174, 1035 (2006).

[23] R. D. Vale, T. Funatsu, D. W. Pierce, L. Romberg, Y. Harada, and T. Yanagida, Direct Observation of Single Kinesin Molecules Moving Along Microtubules, Nature (London) 380, 451 (1996).

[24] G. J. Hoeprich, A. R. Thompson, D. P. McVicker, W. O. Hancock, and C. L. Berger, Kinesin's Neck-Linker Determines Its Ability to Navigate Obstacles on the Microtubule Surface, Biophys. J. 106, 1691 (2014).

[25] E. Muto, H. Sakai, and K. Kaseda, Long-Range Cooperative Binding of Kinesin to a Microtubule in the Presence of ATP, J. Cell Biol. 168, 691 (2005).

[26] K. Sekimoto and J. Prost, Elastic Anisotropy Scenario for Cooperative Binding of Kinesin-Coated Beads on Microtubules, J. Phys. Chem. B 120, 5953 (2016).

[27] R. Dixit, J. L. Ross, Y. E. Goldman, and E. L. F. Holzbaur, Differential Regulation of Dynein and Kinesin Motor Proteins by Tau, Science 319, 1086 (2008).

[28] P. Bieling, I. A. Telley, J. Piehler, and T. Surrey, Processive Kinesins Require Loose Mechanical Coupling for Efficient Collective Motility, EMBO Rep. 9, 1121 (2008).

[29] A. Seitz and T. Surrey, Processive Movement of Single Kinesins on Crowded Microtubules Visualized Using Quantum Dots, EMBO J. 25, 267 (2006).

[30] G. T. Shubeita, S. L. Tran, J. Xu, M. Vershinin, S. Cermelli, S. L. Cotton, M. A. Welte, and S. P. Gross, Consequences of Motor Copy Number on the Intracellular Transport of Kinesin-1-Driven Lipid Droplets, Cell 135, 1098 (2008).

[31] A. G. Hendricks, E. Perlson, J. L. Ross, H. W. Schroeder III, M. Tokito, and E. L. F. Holzbaur, Motor Coordination via a Tug-of-War Mechanism Drives Bidirectional Vesicle Transport, Curr. Biol. 20, 697 (2010).

[32] D. Kenneth, J. W. Driver, A. R. Rogers, P. E. Constantinou, and M. R. Diehl, Two Kinesins Transport Cargo Primarily via the Action of One Motor: Implications for Intracellular Transport, Biophys. J. 99, 2967 (2010).

[33] J. M. Scholey, Kinesin-2: A Family of Heterotrimeric and Homodimeric Motors with Diverse Intracellular Transport Function, Annu. Rev. Cell Dev. Biol. 29, 443 (2013).

[34] B. Prevo, P. Mangeol, F. Oswald, J. M. Scholey, and E. J. G. Peterman, Functional Differentiation of Cooperating Kinesin-2 Motors Orchestrates Cargo Import and Transport in C. elegans Cilia, Nat. Cell Biol. 17, 1536 (2015).

[35] M. Castoldi and A. V. Popova, Purification of Brain Tubulin through Two Cycles of Polymerization-Depolymerization in a High-Molarity Buffer, Protein Expression Purification 32, 83 (2003).

[36] T. Korten, B. Nitzsche, C. Gell, F. Ruhnow, C. Leduc, and S. Diez, Fluorescence Imaging of Single Kinesin Motors on Immobilized Microtubules, Methods Mol. Biol. 783, 121 (2011).

[37] O. H. Lowry, N. J. Rosebrough, A. L. Farr, and R. J. Randall, Protein Measurement with the Folin Phenol Reagent, J. Biol. Chem. 193, 265 (1951).

[38] https://github.com/dmmiedema/Correlation-Imaging.

[39] F. Oswald, A. Varadarajan, H. Lill, E. J. G. Peterman, and Y. J. Bollen, MreB-Dependent Organization of the E. coli Cytoplasmic Membrane Controls Membrane Protein Diffusion, Biophys. J. 110, 1139 (2016).

[40] P. Mangeol, B. Prevo, and E. J. G. Peterman, KymographClear and KymographDirect: Two Tools for the Automated Quantitative Analysis of Molecular and Cellular Dynamics Using Kymographs, Mol. Biol. Cell 27, 1948 (2016). 